\title{
Blasenkarzinom: Risikofaktoren für lokoregionäres Versagen
}

\section{Welche Rolle spielen die lokoregio- nären Rezidivmuster nach radikaler Zystektomie beim Blasenkarzinom und gibt es assoziierte Faktoren?}

D is zu $20 \%$ der Patienten mit muskelinvasivem Blasenkarzinom erleiden lokoregionäre Rezidive, die das Überleben verkürzen. Weil eine adjuvante Therapie das Überleben verbessern könnte, wurden die mit dem Rezidivrisiko asso-

ziierten Faktoren untersucht. 334 Patienten mit Blasenkarzinom (T3-4 N0-1) unterzogen sich einer radikalen Zystektomie und einer Lymphadenektomie im Becken mit oder ohne adjuvante Chemotherapie.

Die Rate für das Gesamtüberleben lag nach einem Jahr bei $63 \%$, nach zwei Jahren bei $43 \%$ und nach drei Jahren bei $39 \%$. Lokoregionäre Rezidive wurden nach einem Jahr bei $16 \%$ der Patienten,

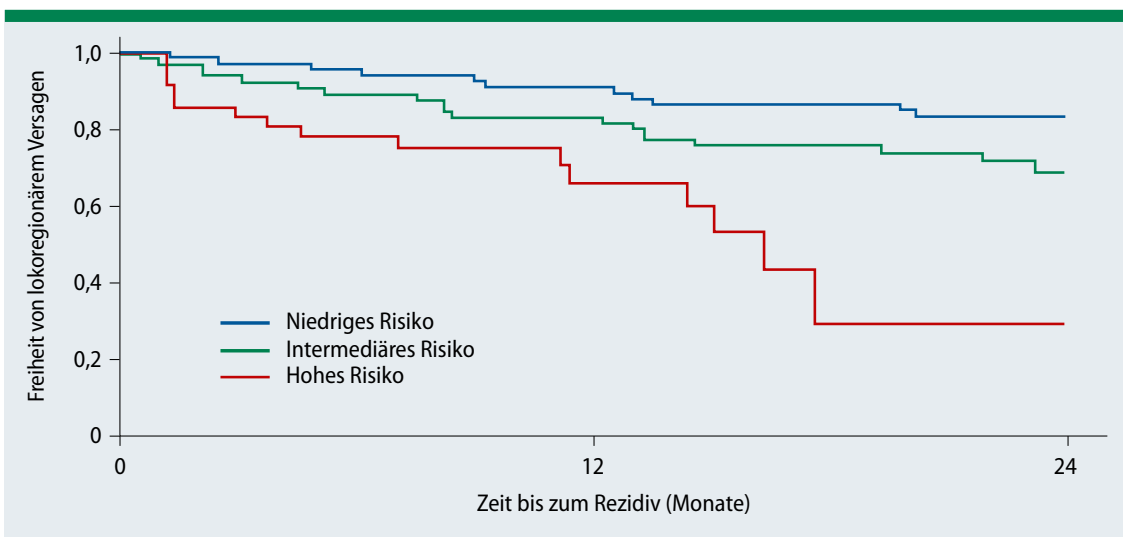

Abb. 1: Lokoregionäres Versagen innerhalb von zwei Jahren bei Blasenkarzinompatienten abhängig von der Risikoeinstufung

\section{Urethrakarzinom: neoadjuvant oder adjuvant?}

\section{Das primäre Urethrakarzinom ist selten und wenig erforscht. Dies be- trifft auch den optimalen Zeitpunkt der perioperativen Chemotherapie.}

A it circa 650 neuen Fällen pro Jahr in Europa gehört das Harnröhrenkarzinom zwar zu den seltenen Krebserkrankungen, endet aber, wenn bereits Lymphknoten befallen sind, nicht selten tödlich. Nach alleiniger Operation liegt die 5-Jahres-Überlebensrate bei rund $40 \%$. Eine platinbasierte Polychemotherapie kann die Prognose auch bei positivem Nodalstatus verbessern, der optimale Zeitpunkt dafür ist jedoch unklar.

Multizentrisch und retrospektiv wurden die Daten von 124 Patienten ausgewertet, die zwischen 1993 und 2012 we- gen eines nicht fernmetastasierten Urethrakarzinoms operiert worden waren.

39 der 124 Patienten (31\%) erhielten eine perioperative Chemotherapie: in zwölf Fällen (31\%) eine neoadjuvante Chemo-(NAC), in sechs Fällen (15\%) eine neoadjuvante Chemoradio-(NCRT) plus adjuvante Chemotherapie $(\mathrm{ACH})$ und in 21 Fällen (54\%) eine adjuvante Chemotherapie. 26 Patienten (21\%) hatten eine lokal fortgeschrittene Erkrankung im Stadium $\geq$ cT3 und/ oder $\mathrm{cN}+$. Von ihnen erhielten 16 (62\%) eine perioperative Therapie.

Die objektiven Ansprechraten auf NAC und N-CRT lagen bei $25 \%$ und $33 \%$. Die 3-Jahres-Überlebensrate bei Patienten mit objektivem Ansprechen (komplett oder partiell) auf die neoadju- nach zwei Jahren bei $28 \%$ und nach drei Jahren bei $31 \%$ beobachtet, am häufigsten in externen/internen iliakalen und Obturator-Lymphknoten.

In der univariaten Analyse waren Resektionsstatus, $\mathrm{pT}$ - und $\mathrm{pN}$-Stadium sowie das Geschlecht mit lokoregionären Rezidiven assoziiert $(\mathrm{p}<0,05)$. In der multivariaten Analyse blieben nur pTund pN-Stadium signifikant (Hazard Ratio 1,92 bzw. 2,57). Drei Risikogruppen wurden definiert: Patienten mit niedrigem (pT3N0), mit mittlerem (pT3N1 oder pT4N0) und mit hohem Risiko (pT4N1) (Abb. 1): Die kumulativen 2-Jahres-Raten des lokoregionären Versagens betrugen hier $15 \%$, $31 \%$ und $72 \%$.

Fazit: Etwa ein Drittel der Patienten mit pT4- oder N1-Blasentumor entwickelt den Studienautoren zufolge innerhalb von zwei Jahren lokoregionäre Rezidive. Sie nehmen an, dass diese Patienten wahrscheinlich von adjuvanten Therapien profitieren würden. Judith Neumaier

Reddy AV et al. Patterns of failure after radical cystectomy for pT3-4 bladder cancer: implications for adjuvant radiation therapy. Int J Radiat Oncol Biol Phys. 2016;94(5):1031-9.

vante Therapie lag bei $100 \%$, bei den übrigen bei $58,5 \%(\mathrm{p}=0,30)$. Bei den $26 \mathrm{~Pa}$ tienten im Stadium $\geq$ cT3 und/oder $\mathrm{cN}+$, die NAC $(n=5), N-C R T(n=3)$, alleinige Operation $(\mathrm{n}=10)$ oder Operation plus $\mathrm{ACH}(\mathrm{n}=8)$ erhalten hatten, lag sie bei $100 \%, 100 \%, 50 \%$ und $20 \%(\mathrm{p}=0,016)$. Die neoadjuvante Therapie war hier mit einem signifikant besseren rezidivfreien $(\mathrm{p}=0,022)$ und Gesamtüberleben nach drei Jahren $(p=0,022)$ verbunden.

Fazit: In der retrospektiven Analyse war die neoadjuvante Therapie gegenüber der sofortigen Operation mit oder ohne adjuvante Chemotherapie mit besserem Überleben assoziiert. Kathrin von Kieseritzky

Gakis $\mathrm{G}$ et al. Impact of perioperative chemotherapy on survival in patients with advanced primary urethral cancer: results of the international collaboration on primary urethral carcinoma. Ann Oncol. 2015; 26(8):1754-9. 